\title{
Hypergraphs with independent neighborhoods
}

\author{
Tom Bohman ${ }^{1, *} \quad$ Alan Frieze $^{1, \dagger} \quad$ Dhruv Mubayi ${ }^{1,2, \ddagger} \quad$ Oleg Pikhurko ${ }^{1, \S}$
}

June 22, 2009

\begin{abstract}
For each $k \geq 2$, let $\rho_{k} \in(0,1)$ be the largest number such that there exist $k$-uniform hypergraphs on $n$ vertices with independent neighborhoods and $\left(\rho_{k}+o(1)\right)\left(\begin{array}{l}n \\ k\end{array}\right)$ edges as $n \rightarrow \infty$. We prove that $\rho_{k}=1-2 \log k / k+\Theta(\log \log k / k)$ as $k \rightarrow \infty$. This disproves a conjecture of Füredi and the last two authors.
\end{abstract}

\section{Introduction}

The neighborhood $N(S)$ of a $(k-1)$-set $S$ in a $k$-uniform hypergraph (henceforth a $k$-graph) is the set of vertices $v$ such that $S \cup\{v\}$ is an edge. For $n \geq k \geq 2$, let $f(n, k)$ be the maximum number of edges in a $k$-graph on $n$ vertices such that all its neighborhoods are independent sets (that is, span no edge). Mantel proved in 1907 that $f(n, 2)=\left\lfloor n^{2} / 4\right\rfloor$, and this was the first result in extremal graph theory. Thus the problem of computing $f(n, k)$ is a natural generalization of Mantel's result.

A $k$-graph is odd if it has a vertex partition $X \cup Y$ such that all edges have an odd number of points less than $k$ in $Y$. It is easy to see that all neighborhoods in an odd $k$-graph are independent sets. Let $b(n, k)$ be the maximum number of edges in an odd $k$-graph. Then the previous observation implies that $f(n, k) \geq b(n, k)$. It was conjectured in [8] that there exists some function $n_{0}(k)$ such that $n>n_{0}(k)$ implies

$$
f(n, k)=b(n, k) .
$$

There was some evidence for this, as it reduces to Mantel's theorem for $k=2$, and it was proved for $k=3$ by Füredi, Pikhurko, and Simonovits [9, 10], thereby settling a conjecture of Mubayi and Rödl [18]. Recently, (1) has also been proved for $k=4$ [8]. As we will show here, (1) is not that far from the truth for $k=5$.

\footnotetext{
${ }^{1}$ Department of Mathematical Sciences, Carnegie Mellon University, Pittsburgh, PA 15213

${ }^{2}$ Department of Mathematics, Statistics, and Computer Science, University of Illinois, Chicago, IL 60607

${ }^{*}$ Research supported in part by NSF grant DMS-0701183

${ }^{\dagger}$ Research supported in part by NSF grant CCR-0502793

${ }^{\ddagger}$ Research supported in part by NSF grant DMS 0653946

${ }^{\S}$ Research supported in part by NSF grant DMS-0457512
} 
Since exact results are rare in extremal hypergraph theory, one often studies asymptotics. In this case, we can define $\rho_{k}=\lim _{n \rightarrow \infty} f(n, k) /\left(\begin{array}{l}n \\ k\end{array}\right)$ which is easily shown to exist [12]. Now conjecture (1) implies that $\rho_{k}=1 / 2$ for all even $k$ and $\rho_{k} \uparrow 1 / 2$ as $k \rightarrow \infty$ for odd $k$. Thus a weaker statement than (1) would be that $\rho_{k}=\lim _{n \rightarrow \infty} b(n, k) /\left(\begin{array}{l}n \\ k\end{array}\right)$, and an even weaker statement is that $\rho_{k} \rightarrow 1 / 2$ as $k \rightarrow \infty$.

In this paper we show that conjecture (1) is false for all $k \geq 7$, and in fact that $\rho_{k} \rightarrow 1$. This follows from an old construction of Kim and Roush [16] which gives lower bounds for the Turán problem for complete $k$-graphs. Thus the small cases shed little light on the behavior of $\rho_{k}$.

We are able to obtain rather sharp estimates on the rate at which $\rho_{k}$ converges to 1 :

Theorem 1. As $k \rightarrow \infty$, we have

$$
1-\frac{2 \log k}{k}+(1+o(1)) \frac{\log \log k}{k} \leq \rho_{k} \leq 1-\frac{2 \log k}{k}+(5+o(1)) \frac{\log \log k}{k},
$$

where $\log$ denotes the natural logarithm. Furthermore, for $k \geq 7$, we have $\rho_{k}>1 / 2$, hence (1) is false for $k \geq 7$.

This leaves open the cases $k=5$ and 6 , where we believe that (1) still holds.

Conjecture 1. $f(n, k)=b(n, k)$ for $k \in\{5,6\}$ and $n$ sufficiently large.

We will present the lower bounds in Theorem 1 via constructions in the next section. Sections 3 and 4 are devoted to the proof of the upper bound. In Section 5 we prove that $40 / 81=0.493 \ldots \leq \rho_{5}<0.534$. We close with some concluding remarks and related open problems.

We associate a $k$-graph with its edge set. For a vertex subset $S$ of size $k-1$, let $d(S)=$ $|N(S)|$. Let $\left(\begin{array}{l}V \\ k\end{array}\right)=\{X \subset V:|X|=k\}$. We denote $[n]=\{1, \ldots, n\}$. Let $\operatorname{Bin}(k, p)$ denote the binomial distribution with parameters $k$ and $p$. In Sections $2-4$, the asymptotic notation $(O(1), o(1)$, etc) will refer to the case when $k$ is fixed and $n \rightarrow \infty$.

\section{Construction}

In this section we prove the lower bound in Theorem 1 by means of a construction due to Kim and Roush. As we will mention in Section 6, this is not the only construction that can be used for this result, but it appears to be the simplest one.

Construction 1 (Kim and Roush [16]). Let $Y_{1} \cup \ldots \cup Y_{l}$ be a partition of $[n]$ into sets, each of size $\lfloor n / l\rfloor$ or $\lceil n / l\rceil$. Let the $k$-graph $\mathcal{H}$ consist of all $k$-sets that have at least one point 
in each $Y_{i}$. Partition $\mathcal{H}$ into $\mathcal{H}_{1} \cup \cdots \cup \mathcal{H}_{l}$, where

$$
\mathcal{H}_{j}=\left\{S \in \mathcal{H}: \sum_{i=1}^{l} i\left|S \cap Y_{i}\right| \equiv j \bmod l\right\} .
$$

By the Pigeonhole Principle, we may assume that there is an $a \in[l]$ with $\left|\mathcal{H}_{a}\right| \leq|\mathcal{H}| / l$. Now let

$$
\mathcal{F}=\mathcal{H} \backslash \mathcal{H}_{a}
$$

Proposition 1. For any $\delta>0$ there is a $k_{0}=k_{0}(\delta)$ such that for all $k \geq k_{0}$ and all sufficiently large $n$ (i.e. $n>n_{0}(k, \delta)$ ), Construction 1 produces a $k$-graph $\mathcal{F}$ on $n$ vertices with independent neighborhoods such that

$$
|\mathcal{F}|>\left(1-\frac{2 \log k}{k}+(1-\delta) \frac{\log \log k}{k}\right)\left(\begin{array}{l}
n \\
k
\end{array}\right)
$$

Proof. To see that $\mathcal{F}$ has independent neighborhoods, consider a $(k-1)$-set $S$. Then $N(S)$ cannot have a point in each $Y_{i}$ for then $\left\{\sum_{i=1}^{l} i\left|(S \cup\{v\}) \cap Y_{i}\right|: v \in N(S)\right\}$ covers all congruence classes modulo $l$. But then $N(S)$ is an independent set, since every edge of $\mathcal{F}$ has a point in each $Y_{i}$.

Let $k>k_{0}(\delta)$ be fixed and $n \rightarrow \infty$. If $l$ is a fixed function of $k$ then we have

$$
\begin{aligned}
|\mathcal{F}| & \geq\left(1-\frac{1}{l}\right)\left(\left(\begin{array}{l}
n \\
k
\end{array}\right)-l\left(\begin{array}{c}
n-\lfloor n / l\rfloor \\
k
\end{array}\right)\right) \\
& \geq\left[\left(1-\frac{1}{l}\right)\left(1-l(1-1 / l)^{k}\right)+\Theta\left(\frac{1}{n}\right)\right]\left(\begin{array}{l}
n \\
k
\end{array}\right) \\
& =\left(1-\frac{1}{l}-(l-1)(1-1 / l)^{k}+\Theta\left(\frac{1}{n}\right)\right)\left(\begin{array}{l}
n \\
k
\end{array}\right) . .
\end{aligned}
$$

Set $l=\lceil k /((2-\epsilon) \log k)\rceil$, where $\epsilon=\log \log k / \log k$. Then using $(1-1 / l)^{k}<e^{-k / l}$ and $k^{\epsilon}=\log k$, we obtain

$$
|\mathcal{F}| \geq\left(1-\frac{(2-\epsilon) \log k}{k}-\frac{1}{k}+\Theta\left(\frac{1}{n}\right)\right)\left(\begin{array}{l}
n \\
k
\end{array}\right)
$$

This gives the required bound.

Proposition 2. For any $k \geq 7$, we have $\rho_{k}>1 / 2$.

Proof. Let us take $l=3$ in Construction 1. The Inclusion-Exclusion Principle shows that $|\mathcal{H}| /\left(\begin{array}{l}n \\ k\end{array}\right)=1-3 \cdot(2 / 3)^{k}+3 \cdot(1 / 3)^{k}+o(1)$. The right-hand side assumes value $\frac{602}{729}>\frac{3}{4}$ for $k=7$ and, as it is not hard to show, is an increasing function of $k \geq 7$. Since $\mathcal{F}$ contains at least $2 / 3$ edges of $\mathcal{H}$, the proposition follows. 


\section{Lemmas}

This section contains some auxiliary results needed in the proof of the upper bound of Theorem 1. It may be possible to extract the following result from [20] (as pointed out to us by a referee). In any case, we give an independent proof below.

Lemma 1. For every $k \geq 100$ there is an $n_{0}$ such that for all $n, x$, and $y$ with $x+y=n \geq n_{0}$ and $\frac{4 n}{k-1} \leq y \leq \frac{n}{2}$, we have

$$
\max _{0 \leq i \leq k-1} \frac{\left(\begin{array}{c}
x \\
i
\end{array}\right)\left(\begin{array}{c}
y \\
k-i-1
\end{array}\right)}{\left(\begin{array}{c}
n \\
k-1
\end{array}\right)} \leq 5\left(\frac{n}{k y}\right)^{1 / 2}
$$

Proof. Let $n_{0}=n_{0}(k)$ be sufficiently large. Let $p=x / n$ and $q=y / n=1-p$. For $0 \leq i \leq k-1$, let $p_{i}=\left(\begin{array}{c}x \\ i\end{array}\right)\left(\begin{array}{c}y \\ k-i-1\end{array}\right)\left(\begin{array}{c}n \\ k-1\end{array}\right)^{-1}$ and $b_{i}=\left(\begin{array}{c}k-1 \\ i\end{array}\right) p^{i} q^{k-1-i}$. We begin by noting that the hypergeometric distribution (as given by $p_{i}$ ) can be bounded by the binomial distribution (as given by $b_{i}$ ). Consider an experiment in which we choose $k-1$ elements of $[n]$ uniformly at random with replacement. Let $X \subset[n]$ with $|X|=x$, and let $\mathcal{D}$ be the event that the $k-1$ random choices are distinct. Note that $b_{i}$ is the probability that exactly $i$ of our randomly chosen element fall in $X$ and $p_{i}$ is the probability that exactly $i$ of our randomly chosen elements fall in $X$ when we condition on $\mathcal{D}$. Therefore,

$$
p_{i} \leq \frac{b_{i}}{\operatorname{Pr}(\mathcal{D})} \leq \frac{b_{i}}{1-\left(\begin{array}{c}
k-1 \\
2
\end{array}\right) \frac{1}{n}}
$$

Note that $b_{i}<b_{i+1}$ if and only if $i+q<(k-1) p$. Therefore, if we set $i_{0}=\lfloor(k-1) p\rfloor$ and $i_{1}=i_{0}+1$ then $\max _{i} b_{i}=\max \left\{b_{i_{0}}, b_{i_{1}}\right\}$. Since $k \geq 3$ and $y \leq n / 2$ we have $x=n-y \geq \frac{n}{2} \geq \frac{n}{k-1}$

and hence $(k-1) p=(k-1) \frac{x}{n} \geq 1$. Also, $\frac{4 n}{k-1} \leq y$ implies that $i_{0}<k-2$. Consequently, $1 \leq$ $i_{0}<k-2$ and we can apply a standard estimate for binomial coefficients (e.g., Inequality (1.5) in $[2])$ :

$$
b_{i_{0}} \leq\left(\frac{(k-1)}{2 \pi i_{0}\left(k-1-i_{0}\right)}\right)^{1 / 2}\left(\frac{(k-1) p}{i_{0}}\right)^{i_{0}}\left(\frac{(k-1) q}{k-1-i_{0}}\right)^{k-1-i_{0}} .
$$

Now let us estimate each of these three terms.

- Since $k \geq 100$ and $p \geq 1 / 2$ we have $\frac{p}{49} \geq \frac{1}{98} \geq \frac{1}{k-1}$. Therefore $\frac{i_{0}}{k-1} \geq p-\frac{1}{k-1} \geq \frac{48}{49} p$. Also

$$
x\left(k-1-i_{0}\right) \geq x(k-1-(k-1) p)=x(k-1)(1-p)=\frac{x y(k-1)}{n} \geq \frac{y(k-1)}{2} \geq \frac{99}{200} y k .
$$

This gives

$$
\left(\frac{k-1}{2 \pi i_{0}\left(k-1-i_{0}\right)}\right)^{1 / 2} \leq\left(\frac{49}{96 \pi p\left(k-1-i_{0}\right)}\right)^{1 / 2}=\left(\frac{49 n}{96 \pi x\left(k-1-i_{0}\right)}\right)^{1 / 2} \leq \alpha\left(\frac{n}{y k}\right)^{1 / 2}
$$

where

$$
\alpha=\left(\frac{200 \times 49}{96 \times 99 \times \pi}\right)^{1 / 2}
$$


- $(k-1) p \leq i_{0}+1$, so

$$
\left(\frac{(k-1) p}{i_{0}}\right)^{i_{0}} \leq\left(\frac{i_{0}+1}{i_{0}}\right)^{i_{0}}<\mathrm{e}
$$

- Since $q+p=1$, we have $(k-1)(q+p)<k$ and so $(k-1) q<k-(k-1) p \leq k-i_{0}$. Therefore

$$
\left(\frac{(k-1) q}{k-1-i_{0}}\right)^{k-1-i_{0}} \leq\left(\frac{k-i_{0}}{k-1-i_{0}}\right)^{k-1-i_{0}}<\mathrm{e} .
$$

Altogether we obtain

$$
b_{i_{0}} \leq \alpha\left(\frac{n}{y k}\right)^{1 / 2} \times \mathrm{e}^{2}
$$

Now let us do the same for $i_{1}$.

- We have $\frac{i_{1}}{k-1} \geq p$. Also

$$
\begin{aligned}
& x\left(k-1-i_{1}\right) \geq x(k-1-(k-1) p-1)=x(k-1)(1-p)-x= \\
& \frac{x y(k-1)}{n}-x \geq \frac{3 x y(k-1)}{4 n} \geq \frac{3 y(k-1)}{8} \geq \frac{297}{800} y k .
\end{aligned}
$$

This gives

$$
\left(\frac{k-1}{2 \pi i_{1}\left(k-1-i_{1}\right)}\right)^{1 / 2} \leq\left(\frac{1}{2 \pi p\left(k-1-i_{1}\right)}\right)^{1 / 2}=\left(\frac{n}{2 \pi x\left(k-1-i_{1}\right)}\right)^{1 / 2} \leq \beta\left(\frac{n}{y k}\right)^{1 / 2}
$$

where

$$
\beta=\left(\frac{800}{594 \pi}\right)^{1 / 2}
$$

- $(k-1) p \leq i_{1}$

- We have $(k-1) q=k-(k-1) p-1 \leq k-i_{1}$. Therefore

$$
\left(\frac{(k-1) q}{k-1-i_{1}}\right)^{k-1-i_{1}} \leq\left(\frac{k-i_{1}}{k-1-i_{1}}\right)^{k-1-i_{1}}<\mathrm{e} .
$$

Altogether we obtain

$$
b_{i_{1}} \leq \beta\left(\frac{n}{y k}\right)^{1 / 2} \times \mathrm{e} .
$$

Now the lemma follows from (2) since $\alpha \mathrm{e}^{2}, \beta \mathrm{e}<5$.

Lemma 2. For every $k \geq 100$ there is an $n_{0}$ such that for all $n \geq n_{0}$ the following holds. Suppose that we have two families $\mathcal{F}$ and $\mathcal{G}$ of $k$-subsets and $(k-1)$-subsets of $[n]$, respectively, such that $|\mathcal{F}| \geq(1-f)\left(\begin{array}{l}n \\ k\end{array}\right)$ and $|\mathcal{G}| \geq g\left(\begin{array}{c}n \\ k-1\end{array}\right)$. Let $[n]=X \cup Y$ with $x=|X|$ and $y=|Y|$ satisfying $\frac{4 n}{k-1} \leq y \leq \frac{n}{2}$. Suppose that reals $0<f^{\prime}, g^{\prime}<1$ satisfy

$$
g^{\prime} f+f^{\prime} g>f+f^{\prime} g^{\prime}+5 f^{\prime} \sqrt{n / k y} .
$$


Then there is an $i, 0 \leq i \leq k-1$, with

$$
\left|\mathcal{F}_{i}\right|=|\{K \in \mathcal{F}:|K \cap X|=i\}| \geq\left(1-f^{\prime}\right)\left(\begin{array}{c}
x \\
i
\end{array}\right)\left(\begin{array}{c}
y \\
k-i
\end{array}\right)
$$

and

$$
\left|\mathcal{G}_{i}\right|=|\{L \in \mathcal{G}:|L \cap X|=i\}| \geq g^{\prime}\left(\begin{array}{c}
x \\
i
\end{array}\right)\left(\begin{array}{c}
y \\
k-i-1
\end{array}\right)
$$

Proof. Suppose on the contrary that no such $i$ exists. Consider

$$
s=\frac{\left(1-g^{\prime}\right)}{\left(\begin{array}{l}
n \\
k
\end{array}\right)}|\mathcal{F}|+\frac{f^{\prime}}{\left(\begin{array}{c}
n \\
k-1
\end{array}\right)}|\mathcal{G}| \geq\left(1-g^{\prime}\right)(1-f)+f^{\prime} g .
$$

Observe that we always have $\left|\mathcal{F}_{i}\right| \leq\left(\begin{array}{c}x \\ i\end{array}\right)\left(\begin{array}{c}y \\ k-i\end{array}\right)$ and $\left|\mathcal{G}_{i}\right| \leq\left(\begin{array}{c}x \\ i\end{array}\right)\left(\begin{array}{c}y \\ k-1-i\end{array}\right)$. Since for each $i$, either $\mathcal{F}_{i}$ or $\mathcal{G}_{i}$ is small (as defined by $(4),(5)$ ), we have $s \leq \sum_{i=0}^{k} \max \left(a_{i}, b_{i}\right)$, where

$$
\begin{aligned}
& a_{i}=\left(1-g^{\prime}\right)\left(1-f^{\prime}\right) \frac{\left(\begin{array}{c}
x \\
i
\end{array}\right)\left(\begin{array}{c}
y \\
k-i
\end{array}\right)}{\left(\begin{array}{c}
n \\
k
\end{array}\right)}+f^{\prime} \frac{\left(\begin{array}{c}
x \\
i
\end{array}\right)\left(\begin{array}{c}
y \\
k-i-1
\end{array}\right)}{\left(\begin{array}{c}
n \\
k-1
\end{array}\right)} \\
& b_{i}=\left(1-g^{\prime}\right) \frac{\left(\begin{array}{c}
x \\
i
\end{array}\right)\left(\begin{array}{c}
y \\
k-i
\end{array}\right)}{\left(\begin{array}{c}
n \\
k
\end{array}\right)}+f^{\prime} g^{\prime} \frac{\left(\begin{array}{c}
x \\
i
\end{array}\right)\left(\begin{array}{c}
y \\
k-i-1
\end{array}\right)}{\left(\begin{array}{c}
n \\
k-1
\end{array}\right)} .
\end{aligned}
$$

Since

$$
a_{i}-b_{i}=\frac{\left(\begin{array}{c}
x \\
i
\end{array}\right)\left(\begin{array}{c}
y \\
k-i
\end{array}\right)}{\left(\begin{array}{l}
n \\
k
\end{array}\right)} \times\left(1-g^{\prime}\right) f^{\prime} \times\left(-1+\frac{(n-k+1)(k-i)}{k(y-k+i+1)}\right),
$$

there is an $i_{0}$ such that $a_{i} \geq b_{i}$ for $0 \leq i<i_{0}$ and $a_{i} \leq b_{i}$ for $i_{0} \leq i \leq k$. Hence,

$$
s \leq \sum_{i=0}^{i_{0}-1} a_{i}+\sum_{i=i_{0}}^{k} b_{i}
$$

Let $P=\left(\begin{array}{c}n \\ k\end{array}\right)^{-1} \sum_{i=0}^{i_{0}-1}\left(\begin{array}{c}x \\ i\end{array}\right)\left(\begin{array}{c}y \\ k-i\end{array}\right)$ and $P^{\prime}=\left(\begin{array}{c}n \\ k-1\end{array}\right)^{-1} \sum_{i=0}^{i_{0}-1}\left(\begin{array}{c}x \\ i\end{array}\right)\left(\begin{array}{c}y \\ k-i-1\end{array}\right)$. Let us choose a random $(k-1)$-subset $L$ of $[n]$ and then let $K$ be obtained from $L$ by adding a random vertex $x \notin L$. Then $K$ is also uniformly distributed. Note that $P$ (resp. $P^{\prime}$ ) is the probability that $K$ (resp $L)$ has less than $i_{0}$ vertices in $X$. Since $L \subset K, P \leq P^{\prime}$. On the other hand $P^{\prime}-P$ is exactly the probability that $x \in X$ and $|L \cap X|=i_{0}-1$. It follows from Lemma 1 that $\operatorname{Pr}\left(|L \cap X|=i_{0}-1\right) \leq 5 \sqrt{n / k y}$ and so $P^{\prime}-P \leq 5 \sqrt{n / k y}$. Hence,

$$
\begin{aligned}
s & \leq P\left(1-g^{\prime}\right)\left(1-f^{\prime}\right)+P^{\prime} f^{\prime}+(1-P)\left(1-g^{\prime}\right)+\left(1-P^{\prime}\right) f^{\prime} g^{\prime} \\
& \leq P\left(1-g^{\prime}\right)\left(1-f^{\prime}\right)+P f^{\prime}+(1-P)\left(1-g^{\prime}\right)+(1-P) f^{\prime} g^{\prime}+5 f^{\prime} \sqrt{n / k y} \\
& =1-g^{\prime}+f^{\prime} g^{\prime}+5 f^{\prime} \sqrt{n / k y} .
\end{aligned}
$$

From (6), we obtain that

$$
\left(1-g^{\prime}\right)(1-f)+f^{\prime} g \leq s \leq 1-g^{\prime}+f^{\prime} g^{\prime}+5 f^{\prime} \sqrt{n / k y}
$$

and this contradicts (3). 


\section{The Upper Bound on $\rho_{k}$}

Before embarking on the formal proof, let us briefly describe the main idea. Suppose that $\mathcal{F}$ is an $n$ vertex $k$-graph with $\rho\left(\begin{array}{l}n \\ k\end{array}\right)$ edges and independent neighborhoods. We may assume that $k$ is large but fixed and $n \rightarrow \infty$. By simple averaging, there is a $(k-1)$-set $S$ with $d(S)=|N(S)| \geq \rho(n-k+1)$. No $k$-set within $N(S)$ can be in $\mathcal{F}$, since $\mathcal{F}$ has independent neighborhoods. Consequently, we obtain

$$
(1-\rho)\left(\begin{array}{l}
n \\
k
\end{array}\right)=\left(\begin{array}{l}
n \\
k
\end{array}\right)-|\mathcal{F}| \geq\left(\begin{array}{c}
\rho(n-k+1) \\
k
\end{array}\right) .
$$

This yields

$$
1-\rho \geq(1-o(1)) \rho^{k}
$$

and solving for $\rho$ gives the bound $\rho \leq 1-(1+o(1)) \frac{\log k}{k}$. This is where the main term $\frac{\log k}{k}$ comes from.

Now suppose we could find not just one neighborhood of size $(1-o(1)) \rho n$ but we could in fact find $k^{1-o(1)}$ such neighborhoods. No $k$-set in any of these neighborhoods lies in $\mathcal{F}$ so we would (roughly) obtain

$$
(1-\rho)\left(\begin{array}{l}
n \\
k
\end{array}\right)=\left(\begin{array}{l}
n \\
k
\end{array}\right)-|\mathcal{F}| \geq k^{1-o(1)}\left(\begin{array}{c}
\rho(n-k+1) \\
k
\end{array}\right) .
$$

This yields

$$
1-\rho \geq k^{1-o(1)} \rho^{k}
$$

and solving for $\rho$ now yields $\rho \leq 1-(1+o(1)) \frac{2 \log k}{k}$. However, the above calculation is not precise since we have over counted some $k$-sets, namely those that lie in two distinct neighborhoods. Thus the main technical details of the proof are concerned with controlling the total amount of over counting in this inclusion/exclusion calculation. We now begin the formal proof.

Take small $\delta>0$. Let $k \geq k_{0}(\delta) \geq 100$ be sufficiently large. Choose large $n_{0}=n_{0}(k, \delta)$. With foresight, we define

$$
c_{0}=4+\delta \quad c_{1}=5+2 \delta \quad c_{2}=5+3 \delta \quad c_{3}=5+6 \delta .
$$

For brevity of notation, let $\epsilon=\log \log k / \log k$. We will show that for all $k>k_{0}$ we have

$$
\rho_{k}<1-\frac{(2-(5+7 \delta) \epsilon) \log k}{k}=1-\frac{2 \log k}{k}+(5+7 \delta) \frac{\log \log k}{k} .
$$

Suppose that this is false for some $k>k_{0}$. Then for infinitely many $n$, in particular for some $n>n_{0}(k, \delta)$, we can find a $k$-graph $\mathcal{F}$ with vertex set $[n]$ and independent neighborhoods such that

$$
|\mathcal{F}|>\left(1-\frac{\left(2-c_{3} \epsilon\right) \log k}{k}\right)\left(\begin{array}{l}
n \\
k
\end{array}\right)
$$


Define

$$
l=\left\lceil\frac{k}{(\log k)^{c_{0}}}\right\rceil .
$$

Our goal is to find sets $A_{1}, \ldots, A_{l}, B_{1}, \ldots, B_{l} \subset[n]$ such that the following conditions hold.

Condition 1: For every $i \in[l]$, the set $A_{i}$ is independent (with respect to $\mathcal{F}$ ), is disjoint from $B_{i}$, and has size

$$
a=\left\lceil\left(1-\frac{\left(2-c_{1} \epsilon\right) \log k}{k}\right) n\right\rceil
$$

Condition 2: The sets $B_{1}, \ldots, B_{l}$ are pairwise disjoint, each of size

$$
b=\left\lceil\frac{\left(2-c_{2} \epsilon\right) \log k}{k} n\right\rceil .
$$

Indeed, if we have such sets then, for any $1 \leq i<j \leq l$, the set $A_{i} \cap A_{j}$ has at most $n-2 b$ elements because its complement contains $B_{i} \cup B_{j}$ as a subset. Since every $k$-set in $\cup_{i=1}^{l}\left(\begin{array}{c}A_{i} \\ k\end{array}\right)$ is missing from $\mathcal{F}$, we have by a simple version of the Inclusion-Exclusion Principle that

$$
l\left(\begin{array}{l}
a \\
k
\end{array}\right)-\left(\begin{array}{l}
l \\
2
\end{array}\right)\left(\begin{array}{c}
n-2 b \\
k
\end{array}\right) \leq\left(\begin{array}{l}
n \\
k
\end{array}\right)-|\mathcal{F}|<\frac{2 \log k}{k}\left(\begin{array}{l}
n \\
k
\end{array}\right) .
$$

Dividing by $\left(\begin{array}{l}n \\ k\end{array}\right)$ and using $k>k_{0}(\delta)$ and $n>n_{0}(k, \delta)$, we get

$$
(1-\delta)\left(\frac{l}{k^{2-c_{1} \epsilon}}-\frac{l^{2}}{2 k^{4-2 c_{2} \epsilon}}\right) \leq \frac{2 \log k}{k},
$$

which is a contradiction (for $\delta<1$ and $k \geq k_{0}(\delta)$ ).

Before proceeding with an argument that gives the sets $A_{1}, \ldots, A_{l}, B_{1}, \ldots, B_{l}$, we need two observations regarding $(k-1)$-sets of large degree. First, observe that for every $(k-1)$-set $S$, we have

$$
d(S)<\left(1-\frac{\log k-2 \log \log k}{k}\right) n,
$$

for otherwise $\left(\begin{array}{l}n \\ k\end{array}\right)-|\mathcal{F}| \geq\left(\begin{array}{c}d(S) \\ k\end{array}\right)>\frac{1}{2} \frac{\log ^{2} k}{k}\left(\begin{array}{l}n \\ k\end{array}\right)$ which is a contradiction.

We will obtain the sets $A_{i}$ as neighborhoods of $(k-1)$-sets. Our strategy is to use the global lower bound on the number of edges to show that there are many $(k-1)$-sets $S$ with large neighborhoods $d(S)$. We would therefore like to restrict our attention to those $(k-1)$-sets with large neighborhoods. Let $\mathcal{G}$ be the collection of $(k-1)$-sets $S \in\left(\begin{array}{c}{[n]} \\ k-1\end{array}\right)$ such that $d(S) \geq n-b$.

Claim 1. $|\mathcal{G}| \geq 2 \delta \epsilon\left(\begin{array}{c}n \\ k-1\end{array}\right)$.

Proof of Claim. Let $|\mathcal{G}|=g\left(\begin{array}{c}n \\ k-1\end{array}\right)$. We have

$$
\begin{aligned}
& k\left(1-\frac{\left(2-c_{3} \epsilon\right) \log k}{k}\right)\left(\begin{array}{l}
n \\
k
\end{array}\right) \leq k|\mathcal{F}|=\sum_{S \in\left(\begin{array}{l}
{[n]} \\
k-1
\end{array}\right)} d(S) \leq \\
& \quad\left(\begin{array}{c}
n \\
k-1
\end{array}\right)(1-g)\left(1-\frac{\left(2-c_{2} \epsilon\right) \log k}{k}\right) n+g\left(\begin{array}{c}
n \\
k-1
\end{array}\right)\left(1-\frac{\log k-2 \log \log k}{k}\right) n,
\end{aligned}
$$


where the last expression comes from (9). Solving for $g$ yields

$$
g \geq \frac{\left(c_{3}-c_{2}\right) \epsilon-2 k^{2} / n}{1-c_{2} \epsilon+2 \log \log k / \log k}>2 \delta \epsilon .
$$

(We used the facts that $c_{3}-c_{2}=3 \delta$ and $c_{2}>2$ in the last inequality.) This completes the proof of Claim 1.

Now we describe how to inductively construct the sets $A_{i}$ and $B_{i}$. Suppose that we have constructed $A_{1}, \ldots, A_{p}, B_{1}, \ldots, B_{p}$ with $0 \leq p<l$ satisfying Conditions 1 and 2 . Let

$$
y=\left\lfloor\frac{2 n}{(\log k)^{c_{0}-1}}\right\rfloor .
$$

and $x=n-y$. Take an arbitrary partition $[n]=X \cup Y$ with $Y \supset \cup_{j=1}^{p}\left([n] \backslash A_{j}\right)$ and $|Y|=y$, which is possible since each set $[n] \backslash A_{i}$ has $n-a \leq 2 n \log k / k$ elements and $p<l$. Our task now is to construct $A_{p+1}$ and $B_{p+1}$.

For an integer $i$, define

$$
\mathcal{F}_{i}=\{S \in \mathcal{F}:|S \cap X|=i\} \quad \text { and } \quad \mathcal{G}_{i}=\{S \in \mathcal{G}:|S \cap X|=i\} .
$$

Also, let

$$
f=2 \log k / k, \quad g=2 \delta \epsilon, \quad f^{\prime}=\log ^{2+\delta} k / k, \quad g^{\prime}=\delta \epsilon .
$$

A short calculation shows by (10) that (3) holds:

$$
f^{\prime}\left(g-g^{\prime}\right)+\left(g^{\prime} f-f-5 f^{\prime} \sqrt{n / k y}\right)>\frac{\delta \epsilon \log ^{2+\delta} k}{k}-C \frac{\log k}{k}>0,
$$

for some absolute constant $C$. So Lemma 2 implies that there is an $i$ such that $\left|\mathcal{F}_{i}\right| \geq$ $\left(1-f^{\prime}\right)\left(\begin{array}{c}x \\ i\end{array}\right)\left(\begin{array}{c}y \\ k-i\end{array}\right)$ and $\left|\mathcal{G}_{i}\right| \geq \delta \epsilon\left(\begin{array}{c}x \\ i\end{array}\right)\left(\begin{array}{c}y \\ k-i-1\end{array}\right)$.

Let $\lambda=f^{\prime} /(\delta \epsilon)$. Let us show that there is a $(k-1)$-set $T_{0} \in \mathcal{G}_{i}$ such that

$$
\left|Y \backslash N\left(T_{0}\right)\right| \leq \lambda(y-k+i+1)
$$

Suppose on the contrary that no such $T_{0}$ exists. Let us count the number $\gamma$ of pairs $(K, z)$ with $K \in \mathcal{F}_{i}$ and $z \in K \cap Y$ in two different ways. On the one hand, we can first choose $K$ and then $z$. This gives

$$
(k-i)\left(1-f^{\prime}\right)\left(\begin{array}{c}
x \\
i
\end{array}\right)\left(\begin{array}{c}
y \\
k-i
\end{array}\right) \leq(k-i)\left|\mathcal{F}_{i}\right|=\gamma .
$$

On the other hand, we can first choose $K-\{z\}$ and then $z$. The set $K-\{z\}$ is either in $\mathcal{G}_{i}$ or not. Taking both cases into account yields

$$
\gamma<\left|\mathcal{G}_{i}\right|(1-\lambda)(y-k+i+1)+\left(\left(\begin{array}{c}
x \\
i
\end{array}\right)\left(\begin{array}{c}
y \\
k-1-i
\end{array}\right)-\left|\mathcal{G}_{i}\right|\right)(y-k+i+1) .
$$

It follows that

$$
\lambda\left|\mathcal{G}_{i}\right|(y-k+i+1)<f^{\prime}\left(\begin{array}{c}
x \\
i
\end{array}\right)\left(\begin{array}{c}
y \\
k-i
\end{array}\right)(k-i) .
$$


Since $\left|\mathcal{G}_{i}\right| \geq \delta \epsilon\left(\begin{array}{l}x \\ i\end{array}\right)\left(\begin{array}{c}y \\ k-i-1\end{array}\right)$, this contradicts the choice of $\lambda$.

Choose an arbitrary set $B_{p+1} \subset X$ that contains all of $X \backslash N\left(T_{0}\right)$ and such that $\left|B_{p+1}\right|=b$. (This is possible because $|X| \geq n-l b \geq b$ and $T_{0} \in \mathcal{G}$, so $\left|X \backslash N\left(T_{0}\right)\right| \leq n-d\left(T_{0}\right) \leq b$.) For every $j \in[p]$, the set $B_{j} \subset Y$ is disjoint from $B_{p+1} \subset X$, so Condition 2 holds. Let $Z=Y \backslash N\left(T_{0}\right)$ and $A^{\prime}=[n] \backslash\left(B_{p+1} \cup Z\right)$. Note that $A^{\prime}$, as a subset of $N\left(T_{0}\right)$, is an independent set. Moreover, by the definition of $T_{0}$ (i.e. by (11)), we have

$$
\left|A^{\prime}\right| \geq n-b-\lambda y \geq n-\left\lceil\frac{\left(2-c_{2} \epsilon\right) \log k}{k} n\right\rceil-\frac{\log ^{2+\delta} k}{\delta \epsilon k} \times \frac{2 n}{(\log k)^{c_{0}-1}} \geq a .
$$

Let us take for $A_{p+1}$ an arbitrary $a$-subset of $A^{\prime}$. Condition 1 clearly holds, finishing the proof.

\section{$5 \quad k=5$}

As we have mentioned in Conjecture 1 , the cases $k=5,6$ remain interesting open questions. By suitably modifying the proof that $\rho_{4}=1 / 2$ from [8], we can obtain fairly good bounds for $\rho_{5}$.

Theorem 2. $0.493 \leq \rho_{5} \leq 0.534$.

Proof. (Sketch) Suppose that $\mathcal{G}$ is a 5 -graph with independent neighborhoods and $\pi\left(\begin{array}{l}n \\ 5\end{array}\right)$ edges which is maximum possible with this restriction. Let $I$ be the 5 -graph

$$
\{12345,12346,12347,12348,12349,56789\} \text {. }
$$

Then a 5-graph with independent neighborhoods is precisely one with no copy of $I$. Consequently, $\mathcal{G}$ contains no copy of $I$. Since $I$ has the property that every two of its vertices lie in an edge, we conclude that if we duplicate any vertex of $\mathcal{G}$ then the resulting 5 -graph also contains no copy of $I$. Now if there are vertices $u, v \in \mathcal{G}$ and any small positive $\epsilon>0$ such that $d(u)>d(v)+\epsilon n^{4}$, then we could delete $v$ and duplicate $u$ to obtain another 5-graph $\mathcal{G}^{\prime}$ with $n$ vertices, independent neighborhoods, and more edges than $\mathcal{G}$ (such a process is sometimes called Zykov symmetrization). This contradiction shows that we may assume all vertex degrees of $\mathcal{G}$ are $(\pi+o(1))\left(\begin{array}{l}n \\ 4\end{array}\right)$.

Now let $A$ be a neighborhood of maximum size, say $|A|=\alpha n$, and $B=[n] \backslash A$. Let $h_{i}$ be the number of edges of $\mathcal{G}$ with exactly $i$ points in $B$; note that $h_{0}=0$ by our hypothesis. Let $\sigma_{i}$ be the sum, over all 4 -sets $S$ with $i$ points in $B$ and $4-i$ points in $A$, of $d(S)$. Then one 
obtains

$$
\begin{gathered}
\left(\begin{array}{c}
\alpha n \\
3
\end{array}\right)(1-\alpha) n \times \alpha n \geq \sigma_{1}=4 h_{1}+2 h_{2} \\
\left(\begin{array}{c}
\alpha n \\
2
\end{array}\right)\left(\begin{array}{c}
(1-\alpha) n \\
2
\end{array}\right) \times \alpha n \geq \sigma_{2}=3 h_{2}+3 h_{3} \\
\alpha n\left(\begin{array}{c}
(1-\alpha) n \\
3
\end{array}\right) \times \alpha n \geq \sigma_{3}=2 h_{3}+4 h_{4} .
\end{gathered}
$$

On the other hand, using the fact that all degrees are almost equal we get

$$
(1-\alpha) n \times(\pi+o(1))\left(\begin{array}{l}
n \\
4
\end{array}\right)=\sum_{x \in B} d(x)=h_{1}+2 h_{2}+3 h_{3}+4 h_{4}+5 h_{5} .
$$

Now consider $3 / 4 \times(12)+1 / 6 \times(13)+1 / 4 \times(14)+(15)$, observe that $\sum_{i=1}^{5} h_{i}=|\mathcal{G}|$, and divide by $n^{5}$. This gives that, as $n \rightarrow \infty$,

$$
\pi \leq \frac{\alpha}{5 \alpha-1}\left(15(1-\alpha) \alpha^{3}+5(1-\alpha)^{2} \alpha^{2}+5(1-\alpha)^{3} \alpha\right)+o(1) .
$$

Maximizing this function over all $\alpha \in(0.5,1)$ yields $\pi<0.534$ and hence $\rho_{5}<0.534$.

For the lower bound, observe that $b(n, 5)=\left(\frac{40}{81}+o(1)\right)\left(\begin{array}{l}n \\ 5\end{array}\right)$ (take $\left.|Y|=\left(\frac{1}{3}+o(1)\right) n\right)$. This shows that $\rho_{5} \geq \frac{40}{81}>0.493$.

\section{Concluding Remarks and Open Problems}

- Our results are similar in flavor to the following problem about the Turán numbers of complete hypergraphs. Let $t_{k}$ denote the maximum proportion of edges in a $k$-graph on $n$ vertices, as $n \rightarrow \infty$, that contains no copy of the complete $k$-graph on $k+1$ vertices. Thus $t_{2}=1 / 2$ by Mantel's theorem. The most famous conjecture in this area, due to Turán [24], is that $t_{3}=5 / 9$, which is achieved by (among others) the 3 -graph with vertex partition $Y_{1}, Y_{2}, Y_{3}$ into almost equal parts and all edges with two points in $Y_{i}$ and one point in $Y_{i+1}$ (indices modulo 3 ) or one point in each $Y_{i}$. Perhaps just as interesting is to determine the growth rate of $t_{k}$ as $k \rightarrow \infty$. Frankl and Rödl [7] proved that $1-t_{k}=O(\log k / k)$ via a construction that has similarities to Construction 1 in this paper. On the other hand, the known upper bound is $t_{k}=1-\Omega(1 / k)$, where the best results are due to Chung and $\mathrm{Lu}$ [3]. It would be very interesting to obtain sharper estimates for $t_{k}$. Perhaps the methods of this paper can be used to show that $1-t_{k}=\omega(1 / k)$, an open question for whose solution de Caen [4, Page 190] offered 500 Canadian dollars.

- For $2 \leq m \leq k$ let the book $B_{k, m}$ be the $k$-graph with the following $m+1$ edges: $[k-$ 1] $\cup\{k+i-1\}$ for $i \in[m]$, and $\{k, k+1, \ldots, 2 k-1\}$. The problem of computing the Turán function $\operatorname{ex}\left(n, B_{k, m}\right)$ has been actively studied $[1,5,6,8,9,10,14,18,21,23]$. Clearly, the 
property not containing $B_{k, k}$ as a subgraph is equivalent to having empty neighborhoods, so $f(n, k)=\operatorname{ex}\left(n, B_{k, k}\right)$. Our results can be modified to show, for example, that for any function $m=m(k)<c_{1} \log k$, where $c_{1}$ is a constant, we have

$$
\pi\left(B_{k, k-m}\right)=1-\Theta\left(\frac{\log k}{k}\right)
$$

as $k \rightarrow \infty$, where $\pi(F)=\lim _{n \rightarrow \infty} \operatorname{ex}(n, F) /\left(\begin{array}{l}n \\ k\end{array}\right)$ denotes the Turán density of a $k$-graph $F$. Indeed, the upper bound on $\pi\left(B_{k, k-m}\right)$ follows from Theorem 1 and the trivial observation that $\operatorname{ex}\left(n, B_{k, k}\right) \geq \operatorname{ex}\left(n, B_{k, k-m}\right)$. The lower bound (16) can be obtained by taking the $k$-graph $\mathcal{F}$ of Construction 1 with $l=k / c_{2} \log k$ where $c_{2} \gg \max \left(c_{1}, 1\right)$ and removing those edges of $\mathcal{F}$ that intersect some part $Y_{i}$ in at most $m$ vertices. As $n \rightarrow \infty$, the proportion of edges that we delete is approximately at most

$$
l \times \operatorname{Pr}(\operatorname{Bin}(k, 1 / l)<m) \leq l \mathrm{e}^{-c_{2} \log k / 4}<\frac{1}{k^{2}} .
$$

(We apply the Chernoff bound here, see e.g. [11, Corollary 2.3].) Therefore, the size of the family $\mathcal{F}$ is at least $(1-1 / l)\left(1-1 / k^{2}\right)\left(\begin{array}{l}n \\ k\end{array}\right)$, and (16) follows.

On the other hand, it is easy to show that $\pi\left(B_{k, m}\right)=o(1)$ if $m=o(k)$. Determining the behavior of $\pi\left(B_{k, m}\right)$ for the intermediate values of $m$ is an interesting open problem.

- A related problem which has been studied a fair amount recently (see, e.g., $[13,15,17,22]$ ) is the maximum possible minimum degree (of $(k-1)$-sets) that a $k$-graph can have without containing some fixed configuration. Let $g(n, k)$ denote the maximum minimum degree of a $k$-graph on $n$ vertices with independent neighborhoods. Then it was shown in [19] that the limit $\gamma_{k}=\lim _{n \rightarrow \infty} g(n, k) / n$ exists. It is trivial to see that $\gamma_{k} \leq 1 / 2$ for all $k$, and odd $k$-graphs show that if $k$ is even, we have equality. It would be interesting to determine the behavior of $\gamma_{k}$ for $k$ odd. As with $t_{k}$, the small cases seem difficult. For $k=3$, the construction for $t_{3}$ above minus the edges with one point in each $Y_{i}$ shows that $\gamma_{3} \geq 1 / 3$. In fact, we make the following conjecture.

Conjecture 2. For every $\epsilon>0$, there exists $n_{0}$ such that if $n>n_{0}$ and $\mathcal{G}$ is an $n$-vertex 3 -graph with every pair lying in at least $(1 / 3+\epsilon) n$ edges, then $\mathcal{G}$ contains a neighborhood that is not an independent set. In particular, $\gamma_{3}=1 / 3$.

- Construction 1 has the following generalization. We begin with some definitions that establish the general setting. Let $a, l \geq 2$ be fixed parameters. Consider the digraph $D$ with vertex set $\mathbb{Z}_{a}^{l}$ and an arc from $x=\left(x_{1}, \ldots, x_{l}\right)$ to $y=\left(y_{1}, \ldots, y_{l}\right)$ if and only if there exists a coordinate $k$ such that

$$
y_{i}=\left\{\begin{array}{ll}
x_{i} & \text { if } i \neq k \\
x_{i}-1 & \text { if } i=k
\end{array} .\right.
$$


Note that the out-degree of each vertex is $l$. We say that a subset $X$ of $\mathbb{Z}_{a}^{l}$ is a perfect cover of $D$ if the out-neighborhoods of the elements of $X$ form a partition of $\mathbb{Z}_{a}^{l}$. In other words, the set $X$ is a perfect cover if for every $y \in \mathbb{Z}_{a}^{l}$ there exists a unique $x \in X$ such that the $\operatorname{arc}(x, y)$ (i.e. the arc directed from $x$ to $y$ ) is in $D$. Note that a perfect cover contains $a^{l} / l$ vertices.

Suppose $X$ is a perfect cover of $D$. Let $n$ be large and fix a partition $Y_{1}, \ldots, Y_{l}$ of $[n]$. For each $k$-set $S$ let $y_{S} \in \mathbb{Z}_{a}^{l}$ be the vector $y_{S}=\left(y_{1}, \ldots, y_{l}\right)$ where $y_{i} \equiv\left|S \cap Y_{i}\right| \bmod a$ for $i=1, \ldots, l$. Now we are ready to define our family with independent neighborhoods. Let $\mathcal{F}$ be the collection of $k$-sets $S$ such that $S \cap Y_{i} \neq \emptyset$ for $i=1, \ldots, l$ and $y_{S} \notin X$. We claim that the collection $\mathcal{F}$ has independent neighborhoods. To see this, consider a $(k-1)$-set $T$. Since $X$ is a perfect cover, there exists $x \in X$ such that $\left(x, y_{T}\right)$ is an $\operatorname{arc}$ in $D$. It follows that there exists an index $k$ such that $T \cup\{z\} \notin \mathcal{F}$ for all $z \in Y_{k}$. In other words, the neighborhood of $T$ (in the hypergraph $\mathcal{F}$ ) does not intersect $Y_{k}$. Since every edge in $\mathcal{F}$ intersects $Y_{k}$, it follows that $\mathcal{F}$ has independent neighborhoods.

In order to ensure a lower bound on the cardinality of the collection $\mathcal{F}$, we consider situations where there is a partition of $\mathbb{Z}_{a}^{l}$ into perfect covers $X_{1}, \ldots, X_{l}$. Each $X_{i}$ corresponds to a collection $\mathcal{F}_{i}$. Furthermore, each set $S$ that intersects $Y_{1}, \ldots, Y_{l}$ is excluded from exactly one of the collections $\mathcal{F}_{i}$. Therefore, there is an index $i$ such that $\left|\mathcal{F}_{i}\right|$ is at least $(1-1 / l)$ times the number of $k$-sets $S$ that intersect $Y_{1}, \ldots, Y_{l}$.

Note that Construction 1 is given by this general setting by taking $a=l$ and letting

$$
X_{j}=\left\{x \in \mathbb{Z}_{l}^{l}: \sum_{i=1}^{l} i x_{i}=j\right\} .
$$

For a second example, set $a=2$ and suppose $l=2^{b}$ for some integer $b \geq 2$. Fix a Hamming code $H \subseteq\{0,1\}^{l-1}$; that is, fix a set of strings $H \subseteq\{0,1\}^{l-1}$ with the property that every string in $\{0,1\}^{l-1}$ is either in $H$ or adjacent (in the $(l-1)$-cube) to exactly one element of $H$. Note that

$$
X=\left\{\left(x_{1}, \ldots, x_{l}\right) \in \mathbb{Z}_{2}^{l}:\left(x_{1}, \ldots, x_{l-1}\right) \in H\right\}
$$

is a perfect cover of $\mathbb{Z}_{2}^{l}$. Furthermore the collection $X, X+e_{1}, X+e_{2}, \ldots, X+e_{l-1}$ is a partition

of $\mathbb{Z}_{2}^{l}$ into perfect covers. Thus, the Hamming code gives another construction that achieves the bound given by Construction 1.

\section{Acknowledgments}

The authors thank the referees for their suggestions which helped to improve the presentation. 


\section{References}

[1] B. Bollobás, Three-graphs without two triples whose symmetric difference is contained in a third. Discrete Math., 8 (1974) 21-24.

[2] B. Bollobás, Random Graphs, 2nd Edition, Cambridge University Press, 2001.

[3] F. Chung and L. Lu, An upper bound for the Turán number $t_{3}(n, 4)$, J. Combin. Theory Ser. A, 87 (1999), no. 2, 381-389.

[4] D. de Caen, The current status of Turán's problem on hypergraphs, Extremal problems for finite sets (Visegrád, 1991), Bolyai Soc. Math. Stud., vol. 3, János Bolyai Math. Soc., Budapest, 1994, pp. 187-197.

[5] P. Frankl and Z. Füredi. A new generalization of the Erdős-Ko-Rado theorem. Combinatorica, 3 (1983) 341-349.

[6] P. Frankl and Z. Füredi. Extremal problems whose solutions are the blowups of the small Witt-designs. J. Combin. Th. A, 52 (1989) 129-147.

[7] P. Frankl and V. Rödl, Lower bounds for Turán's problem, Graphs Combin. 1 (1985), no. $3,213-216$.

[8] Z. Füredi, D. Mubayi and O. Pikhurko, Quadruple systems with independent neighborhoods, J. Combin. Theory, Ser. A 115 (2008), no. 8, 1552-1560.

[9] Z. Füredi, O. Pikhurko and M. Simonovits, On triple systems with independent neighborhoods, Combin. Probab. Comput. 14 (2005), 795-813.

[10] Z. Füredi, O. Pikhurko and M. Simonovits, The Turán density of the hypergraph $\{a b c, a d e, b d e, c d e\}$. Electron. J. Combin. 10 (2003), Research Paper 18, 7 pp. (electronic).

[11] S. Janson, T. Luczak and A. Rucinski, Random Graphs. Wiley, 2000.

[12] G. Katona, T. Nemetz and M. Simonovits, On a problem of Turán in the theory of graphs, Mat. Lapok 15 (1964) 228-238.

[13] P. Keevash, A hypergraph regularity method for generalised Turán problems, Random Structures and Algorithms 34 (2009), 123-164.

[14] P. Keevash and D. Mubayi. Stability results for cancellative hypergraphs. J. Combin. Theory Ser. B, 92 (2004) 163-175.

[15] P. Keevash and Y. Zhao, Codegree problems for projective geometries, J. Combin. Theory Ser. B, 97 (2007), 919-928.

[16] K. Kim and F. Roush, On a problem of Turán, Studies in pure mathematics, 423-425, Birkhäuser, Basel-Boston, Mass. 1983. 
[17] D. Mubayi, The co-degree density of the Fano plane, J. Combin. Theory Ser. B, 95 (2005), no. $2,333-337$.

[18] D. Mubayi and V. Rödl, On the Turán number of triple systems, J. Combin. Theory Ser. A, 100 (2002), no. 1, 136-152.

[19] D. Mubayi and Y. Zhao, Codegree density of hypergraphs, J. Combin. Theory Ser. A, 114 (2007), no. 6, 1118-1132.

[20] W. L. Nicholson, On the normal approximation to the hypergeometric distribution. Ann. Math. Statist. 27 (1956), 471-483.

[21] O. Pikhurko. An exact Turán result for the generalized triangle. Combinatorica, to appear.

[22] V. Rödl, A. Ruciński and E. Szemerédi, A Dirac-type theorem for 3-uniform hypergraphs, Combinatorics, Probability and Computing, Combin. Probab. Comput., 2006, vol. 15, no. 1 - 2, pg. $229-251$.

[23] A. F. Sidorenko. The maximal number of edges in a homogeneous hypergraph containing no prohibited subgraphs. Math Notes, 41 (1987) 247-259. Translated from Mat. Zametki.

[24] P. Turán. On an extremal problem in graph theory (in Hungarian). Mat. Fiz. Lapok, 48 (1941) 436-452. 